\title{
Anabases
}

\section{Érasme revisité, ou les nouveaux visages du De ratione studii : le LabEX «Structuration des mondes sociaux (SMS) »}

\section{Corinne Bonnet}

\section{(2) OpenEdition}

\section{Journals}

Édition électronique

URL : http://journals.openedition.org/anabases/3984

DOI : 10.4000/anabases.3984

ISSN : 2256-9421

Éditeur

E.R.A.S.M.E.

\section{Édition imprimée}

Date de publication : 1 octobre 2012

Pagination : 227-231

ISSN : 1774-4296

\section{Référence électronique}

Corinne Bonnet, «Érasme revisité, ou les nouveaux visages du De ratione studii : le LabEX

"Structuration des mondes sociaux (sms) »», Anabases [En ligne], 16 | 2012, mis en ligne le 01 octobre

2015, consulté le 20 octobre 2019. URL : http://journals.openedition.org/anabases/3984 ; DOI :

10.4000/anabases.3984 
Anabases 16 (2012), p. 227-231.

\section{Érasme revisité, ou les nouveaux visages du De ratione studii : le LabEX «Structuration des mondes sociaux (SMS)»}

Corinne Bonnet

EN FÉVRIER 2012, dans le cadre de la seconde vague des LabEx, un projet piloté par l'université de Toulouse II - Le Mirail et intitulé "Structuration des mondes sociaux » a reçu le label «Laboratoire d'excellence (LabEX) ». Le sous-titre du projet peut aider à comprendre que l'équipe ERASME de l'Unité de Recherche PLH (Patrimoine Littérature Histoire, EA 4601), équipe qui a créé Anabases et fait vivre la revue depuis plus de sept ans, y trouve sa place : " réseaux, régulations, dispositifs, territoires ». Avant d'expliquer aux lecteurs d'Anabases comment, délaissant Rotterdam, Erasme a pris quartier à Toulouse, et comment, mettant de côté son art de la correspondance, il s'adonne désormais au SMS, prenons le temps de présenter ce LabEx.

Le LabEx sMs, dont le responsable scientifique est Michel Grossetti, est une vaste entreprise qui regroupe les forces vives des sciences humaines et sociales toulousaines et qui associe des chercheurs provenant de cinq Unités de recherche de l'université de Toulouse II-Le Mirail : le LISST (sociologie, anthropologie, études urbaines), FRAMESPA (histoire, archéologie), le CERTOP (sociologie), Dynamiques Rurales (géographie) et PLH (par le biais de l'équipe ERASME : histoire), ainsi que des équipes implantées dans d'autres établissements du site toulousain : le LASSP (IEP Toulouse, dans le champs des sciences politiques), le Lereps (Université de Toulouse 1, pour l'économie) et l'équipe IODA de l'inRA-Agir (pour l'économie aussi). Des membres de l'irit (Toulouse 3, inP et CNRS, secteur informatique), du LERASS (information et communication) en font également partie. Les configurations ou croisements disciplinaires que ce consortium rend possible sont donc nombreux et prometteurs. Potentiellement, le nombre total de chercheurs concernés par le LabEx n'est pas loin de 400 permanents. Face à une telle 
richesse et diversité, peut-on vraiment mettre en place une dynamique alliant unité et diversité ? C'est le pari majeur de SMS, coordonné institutionnellement par le PRES de Toulouse, qui joue donc le jeu de l'ouverture, de l'interdisciplinarité, tout en énonçant clairement des présupposés scientifiques et méthodologiques forts, destinés à coaguler l'ensemble des opérations. Quels sont-ils ? Comment définir l'identité de SMS et comment l'équipe ERASME, dont l'objet d'étude principal est la réception de l'Antiquité, y trouve-t-elle sa place?

Le LabeEx SMS se fonde sur un ensemble de sciences humaines et sociales (histoire, sociologie, ethnologie, géographie humaine, anthropologie, psychologie sociale, économie politique), qui considèrent que les phénomènes sociaux sont marqués par des processus historiques et donnent lieu à des formes qui ne sont pas réductibles à l'agrégation ou à la composition d'actions individuelles. Les chercheurs rassemblés au sein du projet SMS travaillent en priorité à la construction de données empiriques sur les phénomènes sociaux. L'objectif central de SMS est donc de développer une conception renouvelée des formes de structuration des mondes sociaux en renforçant la convergence déjà largement amorcée depuis plusieurs années entre équipes et chercheurs de sciences humaines et sociales du site de Toulouse. SMs s'intéressera ainsi aux réseaux sociaux, aux processus de production de conventions, de règles, de normes et de standards qui structurent les organisations et les politiques publiques, aux dispositifs matériels qui interviennent dans les interactions sociales, et aux territoires, considérés comme des structures socio-spatiales cristallisant, de façon toujours historiquement déterminée, des réseaux, des dispositifs et des régulations. Par des opérations empiriques de grande ampleur nécessitant des moyens importants, SMS vise à produire une vision réaliste des mondes sociaux et à affiner l'espace théorique et méthodologique d'analyse des différentes formes de structuration des mondes sociaux. Concrètement, ces objectifs seront déclinés tant dans la recherche - avec la mise sur pied d'une série de projets, séminaires, colloques, enquêtes, etc. - que dans l'enseignement et la formation à divers niveaux (surtout Master et Doctorat).

Les mots-clés du projet - réseaux, régulations, dispositifs, territoires - dessinent déjà des orientations majeures au sein desquelles des "opérations " ponctuelles, mais généralement transdisciplinaires, vont se développer. Le LabEx SMS a en effet été doté d'un budget de 7 millions d'euros pour une durée de 8 ans. Cela permettra de financer les diverses entreprises proposées en réponse à l'appel à projets, ainsi que d'autres qui émergeront en cours de route ; il rendra possible l'engagement de doctorants et postdoctorants rattachés à telle ou telle opération de recherche. En raison de l'expérience acquise ces dernières années, c'est dans l'étude des réseaux qu'ERASME apportera sa pierre à l'édifice, en particulier des réseaux intellectuels qui dessinent des territoires scientifiques ou disciplinaires. Quelques rappels ne sont sans doute pas superflus...

PLH (Patrimoine Littérature Histoire - EA 4601) dans son ensemble a pour domaine d'étude l'analyse des rapports et des modes d'articulation entre passé et présent, selon une approche de nature philologique, historique et herméneutique. Les champs des littératures et des sciences historiques y sont appréhendés comme des lieux 
et des enjeux de mémoire, comme des espaces de créativité et d'interaction, selon une méthode transversale et comparatiste entre disciplines, entre aires culturelles et entre périodes, de l'Antiquité au très contemporain. Trois axes s'y dégagent : dresser l'état des lieux des patrimoines culturels, et réfléchir sur les manières dont nos sociétés se les approprient ; étudier les phénomènes de construction, transmission, traduction et transposition des savoirs ; analyser la position des sociétés d'hier et d'aujourd'hui quant à la notion de " patrimoine " et de " mémoire ".

Au sein de PLH, l'équipe ERASME est la seule en France à travailler spécifiquement sur la réception de l'Antiquité. Elle explore les différentes formes de relations dynamiques et critiques qui rendent les mondes anciens contemporains, à travers les articulations entre présent, passé et avenir. On lui doit la création en 2005 de la revue Anabases. Traditions et réceptions de l'Antiquité qui illustre, à raison de deux fascicules par an, la fécondité de cette approche dynamique de l'Antiquité. Parmi les axes de recherche de l'équipe ERASME, on relèvera notamment celui qui porte sur « Le partage des savoirs : historiographie, philosophie, littérature » et celui qui explore la thématique "Réseaux scientifiques et histoire des savoirs sur l'Antiquité (XIX $\mathrm{e}-\mathrm{XX}$ e siècles) ». Partant du postulat que le champ intellectuel, en perpétuel mouvement, repose sur un principe modulable de partage et d'échange des connaissances, au sein d'une communauté qui s'auto-représente sous la forme d'une "République des lettres » idéale, on s'est efforcé de comprendre comment, sur le plan pragmatique, le monde de la production des savoirs se structure, comment les échanges s'organisent, fonctionnent et produisent de la «culture » sous diverses formes. L'exploration de l'économie des réseaux, par le biais notamment des correspondances, se situe ainsi à la croisée de plusieurs approches et disciplines : l'étude des pratiques savantes (ou "sociabilité savante »), l'archéologie des savoirs, l'histoire culturelle, les diverses branches des sciences de l'Antiquité, l'épistolographie, la sociologie des réseaux, l'histoire littéraire, etc., dans un va-et-vient constant entre le passé (l'Antiquité) étudié par ces savants et le présent ( $\mathrm{du} \mathrm{XVI} \mathrm{e}^{\mathrm{e}}$ au XXI ${ }^{\mathrm{e}}$ siècle) dans lequel les réseaux se configurent. Or, dans les réseaux qui structurent le monde savant, l'Antiquité jouit d'un statut particulier. Si, en effet, elle constitue une référence " universelle " pour les élites de tous les pays, de la Renaissance à l'époque contemporaine, elle est aussi, à l'ère de la construction des nations (XVIII $-\mathrm{XIX}^{\mathrm{e}}$ siècles), fortement sollicitée dans le cadre de bricolages identitaires faisant appel à un passé lointain et prestigieux.

Après avoir participé au GDRI "Les mondes lettrés », coordonnée par Christian Jacob (CNRS Paris) et à l'entreprise éditoriale de "Les lieux de savoir ", 1, "Espaces et communautés»(http ://lieuxdesavoir.hypotheses.org/lieux-de-savoir-1), ERASME a organisé plusieurs Colloques, Journées d'étude et Séminaires et a pris part à diverses initiatives touchant à l'archéologie des savoirs et à l'étude des pratiques de sociabilité savante. On citera en particulier :

- le Colloque intitulé « S'écrire et écrire sur l'Antiquité. L'apport des correspondances à l'histoire des travaux scientifiques " (coordonné par C. Bonnet et V. Krings), publié 
en 2008 (Grenoble, 416 p. : http ://www.millon.com/collections/histoire/horos/ecrire. html) ;

- un Séminaire bisannuel (2008/9 et 2009/10) sur le thème "L'Antiquité en réseaux » (coordonné par C. Bonnet, V. Krings et C. Valenti), paru sous le titre Connaître l'Antiquité. Individus, réseaux, stratégies du XVIII au XXI siècle, PUR, Rennes, 2011 (273 pages : http ://www.pur-editions.fr/detail.php ?idOuv=2544);

- une Journée d'étude sur "Les Antiquaires du Midi ", publiée sous forme de livre Les Antiquaires au Midi. Savoirs et mémoires. XVI -XIX siècle (coordonné par V. Krings et C. Valenti), Paris, Errance, 2011 (http ://www.compitum.fr/publications/1869krings-valenti-les-antiquaires-du-midi-savoirs-et-memoires-xvie-xixe-siecle) ;

- le travail mené par Corinne Bonnet sur la correspondance de Franz Cumont (plus de 12000 lettres de correspondance passive), depuis 1995, avec la mise en ligne d'une base de données complète et une série de publications (plusieurs livres et plus de 20 articles) : http ://www.academiabelgica.it/index.php ?id_page=90\&id_ lang=1\&SID\&menu=cumont ;

- un Programme ponctuel de recherche financé par le Conseil Scientifique de l'université de Toulouse II - Le Mirail, en 2009-10 pour réaliser un " Portail informatique sur les correspondances de savants antiquisants francophones des $\mathrm{XIX}^{\mathrm{e}}-\mathrm{XX}$ e siècles ". La première phase du projet est réalisée : http ://w3.epistula.univ-tlse2.fr/userlogin.php.

Dans le sillage de ces activités, ERASME continuera de développer, dans un premier temps, au sein du LabEX sMS, deux projets entamés, qu'il s'agit de développer et d'orienter vers de nouveaux questionnements en rapport avec la «structuration des mondes sociaux ». En effet, au sein de SMS, une opération de recherche portera sur la "Structuration du monde scientifique » et sera animée par Denis Eckert, Michel Grossetti et Corinne Bonnet. De la République des Lettres à Internet, il s'agira d'y réfléchir aux évolutions des " cartes " scientifiques, aux équilibres entre les différents espaces et aux tensions qu'ils révèlent à diverses échelles, du local au global. Dans ce cadre, on approfondira l'apport des Antiquaires du Midi, c'est-à-dire des érudits ou collectionneurs qui se sont intéressés aux antiquités entre le XVI ${ }^{\mathrm{e}}$ et le XIX ${ }^{\mathrm{e}}$ siècle et qui représentent un milieu particulièrement riche dans la France méridionale marquée par l'héritage antique. Ils illustrent une phase de l'histoire savante qui précède la professionnalisation universitaire de l'étude de l'Antiquité. Or, les collections que ces Antiquaires ont réunies, les travaux qu'ils ont réalisés, les réseaux de sociabilité qu'ils ont développés autour de leur goût de l'antique et de leur curiosité polymorphe participent à une fabrique des savoirs dont les savants universitaires de la phase successive seront à la fois les héritiers et les critiques. Par ailleurs, on poursuivra la réalisation du portail informatique "Epistula ", en recensant tous les fonds de correspondance des antiquisants francophones des $\mathrm{XIX}^{\mathrm{e}}$ et $\mathrm{XX}^{\mathrm{e}}$ siècles. Une fois cet outil complété, on pourra, avec l'aide des instruments d'analyse et de cartographie des réseaux sociaux, lancer plusieurs études sur les territoires qui caractérisent et les dynamiques qui animent et configurent le champ des Sciences de l'Antiquité à cette époque. Dans la perspective de L'archéologie du savoir de Foucault, il s'agira d'étudier des " programmes " intellectuels 
relationnels, relevant de dispositifs stratégiques qui régulent le flux des connaissances, déterminent des centres et des périphéries.

Voilà comment, cher Thomas More, je suis embarqué sur une étrange caravelle, toutes voiles déployées, avec un équipage très bigarré, qui semble promettre la découverte de nouveaux mondes... Mon ami Guillaume Budé me conseille de lire son De contemptu rerum fortuitarum et d'avoir confiance dans la translatio studiorum que ce vaste chantier engage. Concedo nulli, ai-je l'habitude de me répéter, "je ne fais de concession à personne "... Il est donc temps d'être à la hauteur de nos ambitions! G bcp de nostalj de vs lol! Votre Erasme.

Corinne BONNET

PLH-ERASME

Pavillon de la recherche

5, allées $A$. Machado

F-31058 Toulouse cedex 9

corinne.bonnet@sfr.fr 